\section{PTEN goes paracrine}

Phosphatase and tensin homologue deleted on chromosome ten (PTEN) is a negative regulator of phosphoinositide-3 kinase ( $\mathrm{PI}(3) \mathrm{K}$ ) signalling and a tumour suppressor. Parsons and colleagues (Science http://doi.org/m7z; 2013) have identified a translational variant of PTEN (PTEN-Long) that similarly antagonizes the $\mathrm{PI}(3) \mathrm{K}$ pathway and suppresses tumour growth, but is secreted and can enter other cells.

The authors found an in-frame alternative translation initiation codon upstream of the canonical AUG in the PTEN transcript, and subsequently detected the $75-\mathrm{kDa}$ PTENLong protein in mouse embryonic stem cells and human cancer cell lines. This extended variant reduced $\mathrm{PI}(3) \mathrm{K}$ signalling in a phosphatase-dependent manner. Computer modelling revealed the presence of a secretion signal sequence within the additional 173 amino acids of PTEN-Long, and the authors accordingly detected endogenous PTEN-Long in human plasma and serum. Although PTEN interacts with heparan-sulphate-modified cell-surface receptors, Parsons and colleagues demonstrated that a poly-arginine stretch present within PTEN-Long facilitated cell penetration, and subsequently showed that purified PTENLong inhibited PI(3)K signalling, an effect that was abrogated by mutating the poly-arginine sequence. Like PTEN, PTEN-Long induced cell death, and treatment with PTEN-Long induced tumour regression in various mouse models. Notably, some cancers were found to contain a mutated form of PTEN-Long with reduced signalling capacity, which might be preferentially selected during tumour development. Whether PTEN-Long has therapeutic potential remains to be investigated.

$\mathrm{KL}$

\section{Spindle positioning by membrane adjustment}

Accurate positioning of the mitotic spindle in the centre of the cells, critical for the generation of equally sized daughter cells, is mediated by a cortical dynein-dynactin complex pulling on astral microtubules. Kiyomitsu and Cheeseman have characterized the anaphase recruitment pathway for cortical dynein and discovered an unexpected role for the plasma membrane in centring the spindle. (Cell http:// dx.doi.org/10.1016/j.cell.2013.06.010; 2013).

The recruitment of dynein-dynactin to the cortex in metaphase is known to require its cortical targeting factor LGN and the LGNbinding protein Gai. However, the authors find that in anaphase, there is an LGN-dependent recruitment pathway that is independent of Gai, but requires $4.1 \mathrm{G}$ and $\mathrm{R}$ proteins and is suppressed by cyclin-dependent kinase (CDK) activity. Blocking both the LGN- and 4.1-dependent pathways for cortical dynein recruitment resulted in unequally sized cells after division, with small cells delaying their cell cycle. Anaphase spindle elongation was also impaired in cells depleted of both LGN and 4.1. Surprisingly, visualization of the plasma membrane during division revealed that in cells with an off-centred spindle, the

\section{Obesity-associated gut microbiota induce liver cancer}

The senescence-associated secreted phenotype (SASP) is known to modulate tumorigenesis. Both secretion of inflammatory cytokines in SASP and gut microbiota changes have been seen in obese mice. Ohtani, Hara and colleagues have discovered that obesity leads to changes in gut microbial metabolites that induces SASP in hepatic stellate cells, the secretion of cancer-inducing inflammatory cytokines and liver tumorigenesis (Nature 499, 97-101; 2013).

The authors used two models of obesity in mice and a chemical carcinogen treatment that induces oncogenic Ras. They observed an increase in DNA damage and senescence in hepatic stellate cells. Strikingly, tumours were reduced in animals deficient for interleukin 1-beta (IL-1 $\beta$ ) and in animals treated with antibiotics. The latter suggested that changes in gut microbiota may have led to the induction of hepatic stellate cell senescence and secretion of inflammatory cytokines. Indeed, the authors found that obese animals showed an increase in the levels of deoxycholic acid (DCA), a bacterial metabolite that is known to induce DNA damage and senescence. This metabolite is mainly produced by bacteria belonging to the Clostridium cluster, which the authors found were increased in the gut microbiota of obese animals. The authors were able to lower the tumorigenic development by using compounds limiting DCA production. However, as for IL-1 $\beta$ deficiency, some tumours persisted, indicating that other factors must contribute to liver tumorigenesis in this setting. plasma membrane elongated in an asymmetric fashion, leading to a correction of spindle position. Membrane blebs forming on the elongating membrane were partly responsible for this elongation, and the authors found a correlation between the proximity of chromatin to the cortex and elongation of the membrane. Further experimentation led the authors to suggest that a chromosome-derived Ran gradient reduces levels of Anillin and other cortical proteins, allowing membrane reorganisation. CKR

\section{Rac1 controls intestinal tumorigenesis}

Mutation of the $A P C$ gene is a common occurrence in colorectal cancer. Sansom and colleagues now report that following Apc loss, RAC1 activity drives proliferation of LGR5positive intestinal stem cells (ISCs) and tumorigenesis through ROS production and NF- $\kappa \mathrm{B}$ signalling (Cell Stem Cell 12, 761-773; 2013).

In line with previous reports, the authors showed that Apc loss led to the Myc-dependent upregulation of Rac guanine exchange nucleotide factors (GEFs), Rac1b and subsequent activation of Rac1. Deletion of Racl in the mouse intestine decreased the hyperproliferation of intestinal crypt progenitors, the expansion of the LGR5-marked ISC population and the upregulation of ISC gene signatures observed in the context of Apc deficiency. Rac1 is known to promote ROS production, and the authors observed that increased ROS levels were required for LGR5-positive cell expansion following Apc loss and RAC1 activation. The NF- $\kappa \mathrm{B}$ pathway, which has been previously linked to ISC expansion, was also found to promote the hyperproliferation of LGR5-marked cells downstream of Rac1. Deletion of Rac1 was further shown to prevent mouse intestinal adenoma formation following Apc loss in the LGR5-positive cell population, and ROS reduction also extended the tumour-free lifespan of these Apc-deficient mice. When induced after tumours were allowed to form, Rac1 deletion reduced the LGR5-positive tumour cell population of Apc-mutant mice.

These findings underscore the importance of Racl activity in intestinal tumorigenesis. AIZ

By Nathalie Le Bot, Katrin Legg, Christina Karlsson Rosenthal and Alexia-Ileana Zaromytidou 